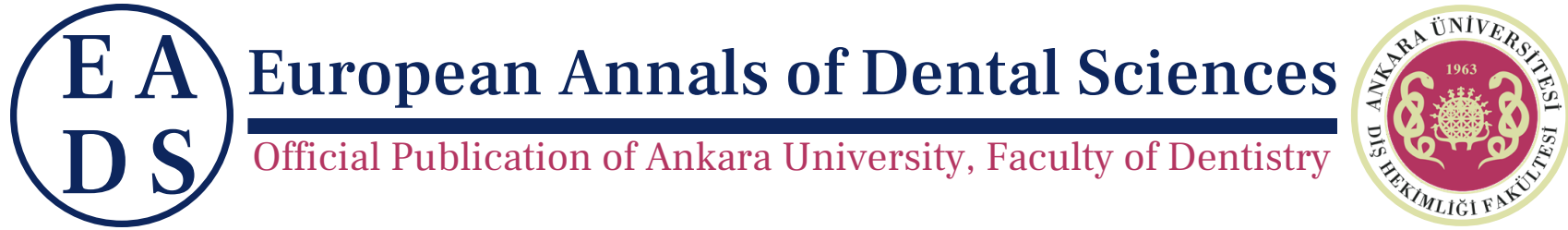

EADS, 2021, 48 (1), 28-32

\title{
Rehabilitation of a Severely Worn Dentition with Partial Lithium-disilicate Glass Ceramic Restorations: A Case Report
}

\author{
Ece İrem Oğuz $\odot$ 1,* and Semih Berksun $\odot 2$ \\ ${ }^{1}$ DDS, Department of Prosthodontics, Ankara University Faculty of Dentistry, Ankara, Turkey. and ${ }^{2}$ DDS, \\ $\mathrm{PhD}$, Department of Prosthodontics, Ankara University Faculty of Dentistry, Ankara, Turkey. \\ *Corresponding Author; eikiyan@ankara.edu.tr
}

\begin{abstract}
Rehabilitation of worn dentition is a challenging task because several predisposing factors may be included in the pathogenesis. The treatment options and process should be carefully considered based on the clinical condition and patients' requirements. This case report represents the esthetic rehabilitation of a 68 -year-old patient who exhibited severely worn teeth caused by attrition and erosion. After clinical evaluation, $3 \mathrm{~mm}$ increase in occlusal vertical dimension was planned with partial lithium-disilicate ceramic restorations as laminate veneers for anterior teeth and vonlays (onlays with veneer extentions) for posterior teeth in maxilla. The adaptation of the patient to the increased occlusal vertical dimension was tested for 1 month by provisional direct composite restorations. As the patient did not show any discomfort, definitive restorations were completed. The patient was satisfied with the esthetics and functionality of her new dentition.
\end{abstract}

Key words: esthetics; laminate veneers; lithium disilicate; occlusal vertical dimension; worn dentition.

\section{Introduction}

Tooth wear can be defined as loss of hard tooth structures which is caused by pathological or physiological processes without the involvement of bacterial action. ${ }^{1,2}$ Physiological tooth wear is a gradual process that depends on the patient's age. Pathological wear can occur as attrition, abrasion, abfraction, and erosion based on the ethiologic factor. ${ }^{2}$ Among these, erosion and attrition may cause fast destruction on hard tooth structures. ${ }^{3}$ Erosion is a chemical process, mainly caused by the consumption of excessive acidic drinks or digestive disorders. ${ }^{4}$ Attrition is caused by functional or parafunctional mastication such as bruxism and results in mechanical wear which can be detected as facets on the contacting surfaces of opposing teeth. ${ }^{2,5}$ The more acidic environment exists in the oral cavity, the more attrition would be encountered. ${ }^{6}$ Dental attrition and erosion are consecutive processes that can lead to excessive damage if not treated properly. ${ }^{5,7}$ Severe tooth wear may cause not only esthetic compromise, but also functional problems due to loss of occlusal vertical dimension (OVD). ${ }^{8}$ The treatment of severely worn dentition might be complicated and troublesome because of the complex etiology. ${ }^{1,7}$ Before pro- ceeding with treatment, patient should be examined very carefully based on the dental and medical anamnesis to identify the causes of tooth wear. It is crucial to eliminate the main reasons of tooth wear prior to prosthodontic intervention. ${ }^{1}$

The treatment plan of worn dentition can vary from preventive and local treatments to full mouth rehabilitations depending on the severity of the situation. ${ }^{2}$ As the concept of minimally invasive dentistry spreads, new dental materials, in combination with advanced adhesives have taken place in the dental market, allowing practitioners to accomplish more esthetic and retentive restorations with the removal of minimal amounts of hard tooth structures. ${ }^{5}$

This case report exhibits the treatment concept of a patient with worn dentition based on conservative and adhesive treatments by means of laminate veneers and ceramic onlays with esthetic veneer extensions, currently called as vonlay.

\section{Case Report}

A 68-year-old female referred to Prosthodontic Clinic of the Ankara University Faculty of Dentistry, due to esthetic concern 


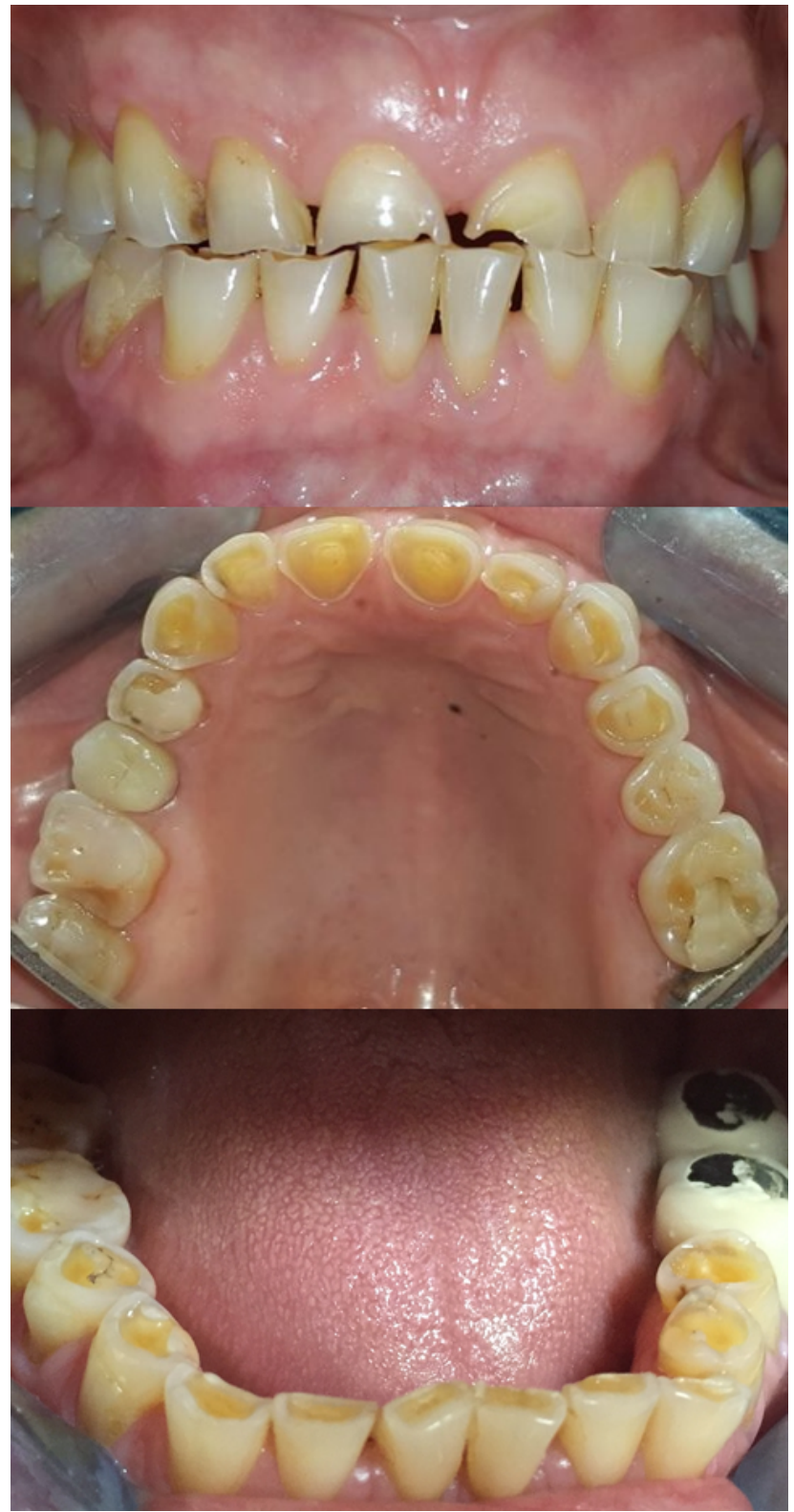

Figure 1. Intraoral photographs of the patient before treatment

about her discolored and severely worn maxillary teeth. She reported that she used to consume excessive amounts of acidic beverages and that she had the habit of grinding her teeth at night for a long time (nocturnal bruxism). During the initial visit, intraoral photographs were taken and radiographic examination was performed. Intraoral examination revealed several composite and amalgam fillings on teeth, porcelain fused to metal (PFM) restorations on teeth 25,36 , and 37 , and severely worn dentition (Figure 1). Root canal treatments were observed on teeth 24 and 25 during the radiographic evaluation (Figure 2).

There were no signs of temporomandibular disorder (TMD) symptoms; however, the patient stated having received occlusal splint therapy based on the pain in TMJ and did not recall the exact time. Oral hygiene was satisfying as she brushed her teeth twice a day without flossing. Treatment options were discussed with the patient. The porcelain fused to metal rehabilitation option was elected as the patient desired the most esthetic and natural look. Full-mouth rehabilitation was suggested, but the patient stated that she had aesthetic considerations for maxillary teeth only and that her mandibular teeth

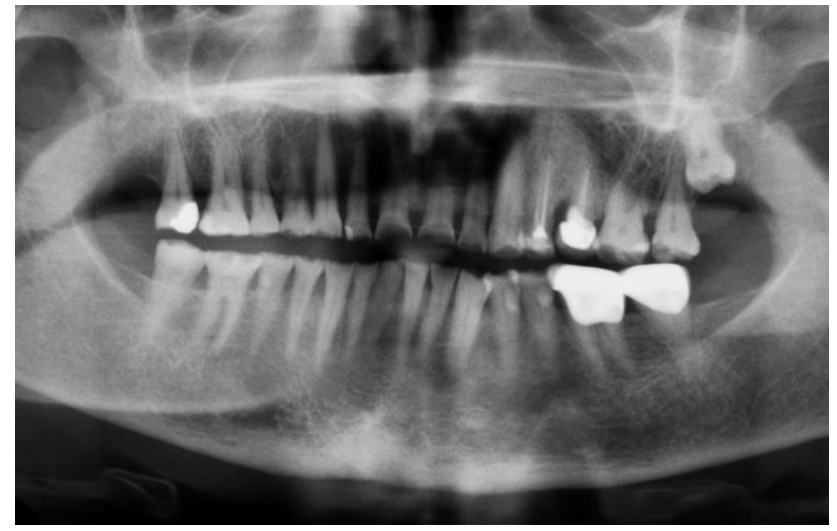

Figure 2. Panoramic radiograph before treatment

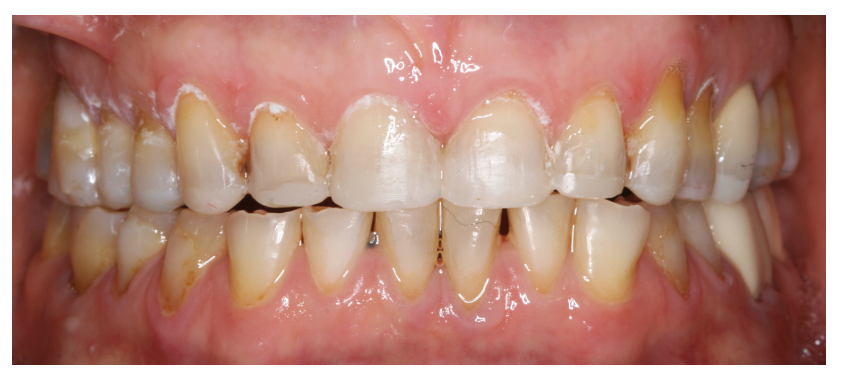

Figure 3. Direct resin composite interim restorations to test newly established OVD

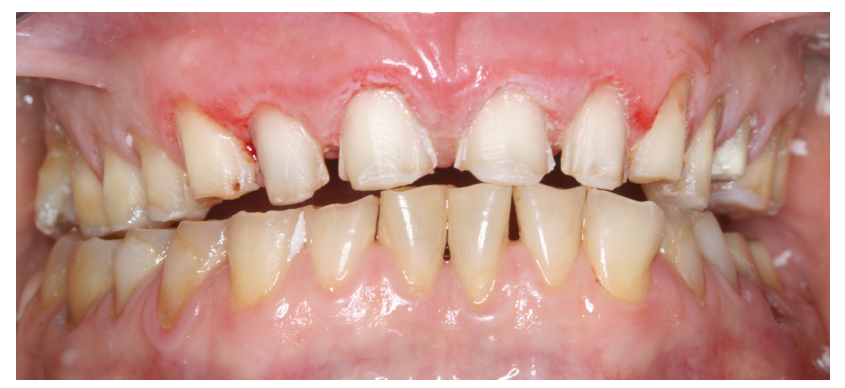

Figure 4. Intraoral view of the tooth preparations

were not visible during smile. Also, based on the financial considerations, she preferred to only receive maxillary esthetic restorations. Although the patient was warned about the condition of the mandibular dentition and the problems that may occur in the future due to the wear, she refused to receive any mandibular treatments except for the defected PFM restorations. Therefore, the treatment plan was settled as maxillary full-mouth lithium disilicate glass-ceramic restorations with minimal preparation.

Clinical evaluation revealed that excessive attrition caused insufficient intermaxillary space and OVD loss. The Niswonger method was used to determine the amount of OVD loss. ${ }^{9}$ The difference in vertical dimension between the resting and occlusion position of mandible should be $2 \mathrm{~mm},{ }^{10}$ yet it was $5 \mathrm{~mm}$ for our patient which referred to $3 \mathrm{~mm}$ loss in OVD. To evaluate the adaptation of the patient to OVD reconstruction, direct resin composite restorations were performed on occlusal surfaces of maxillary posterior teeth and incisal edges of maxillary anterior teeth (Figure 3). After 1 month of a trial period with newly established OVD, the patient was comfortable with the new occlusion, there was no sign of temporomandibular disorder or muscular pain, the facial type was unaffected, and adequate 


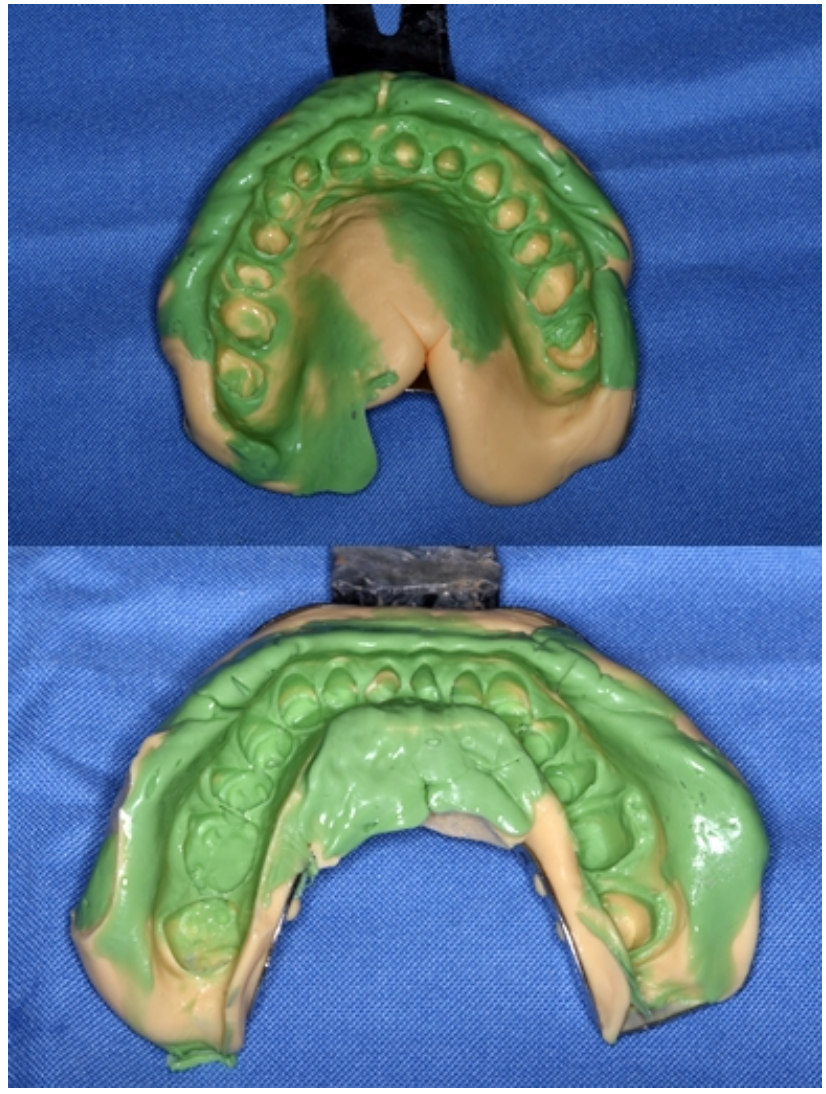

Figure 5. Maxillary and mandibular additional silicone impressions

lip closure was maintained.

Gingivectomy was performed on maxillary anterior teeth to level gingival margins and increase clinical crown height. After the healing period of gingival soft tissue, maxillary anterior teeth were prepared to receive laminate veneers and maxillary posterior teeth were prepared to receive onlay restorations with veneer extentions (Figure 4). For simplicity' sake, we will refer to this restoration as vonlay. A hard tissue reduction of $0.5-0.8$ $\mathrm{mm}$ was obtained for labial and buccal surfaces, and $2 \mathrm{~mm}$ reduction was performed on occlusal and incisal surfaces (Figure 4). The preparations were extended to the palatal $2 / 3$ of teeth with incisal/occlusal overlap as this preparation design was approved to provide the best support for the restorative materials with predictable outcome. ${ }^{11}$ Also, defected PFM restorations on teeth 25,36 , and 37 were removed and teeth were prepared to receive full-covarage restorations.

Retraction cords were placed into every sulcus of the prepared teeth (Ultrapak 00; Ultradent Products Inc., South Jordan, Utah, USA). Then, the impressions were made with a two-step technique using additional silicone material (Elite HD Putty and Light Fast set; Zhermack Dental, Badia Polesine, Italy) (Figure 5). The interocclusal centric record was made at the previously determined vertical dimension and the facebow transfer was completed (Artex; Amann Girrbach AG, Koblach, Austria).

Maxillary definitive restorations were completed with lithium-disilicate glass ceramics (IPS e.max Press HT ingots, A2 shade; Ivoclar Vivadent, Schaan, Liechtenstein) (Figure 6) and mandibular left molars received PFM. All restorations were cemented with a dual-cure resin cement (Variolink N; Ivoclar Vivadent) according to the instructions of the manufacturer. Cementation was performed for each tooth one by one with extreme caution to protect flossable contacts. The patient was pleased with the function and esthetics of the final result (Figures 7 and 8 ). A vacuum-formed night-guard (Duran; ScheuDental Gmbh, Iserlohn, Germany) was manufactured and the patient was notified to wear it during the night to prevent possible restoration fractures. The patient was instructed about oral hygiene and called for regular checkups in every 6 months to maintain oral health.

\section{Discussion}

Tooth wear is a multifactorial condition that may occur due to mechanical and chemical risk factors. ${ }^{12}$ In the present case, both factors were related as the patient stated a history of bruxism and acidic drink consumption. In most cases, patients do not realize the magnitude of their worn dentition until it is too late for preventive treatments. As such, this condition may leave no choice to the clinician but to restore all affected teeth. Even tough full-mouth restorations could have been performed for the present case, the patient demanded not to restore mandibular teeth as her main complaint was with the maxillary dentition. As the increase in OVD and a functional occlusion could be achieved with maxillary restorations only, this situation did not lead to any restorative problems.

The restoration of the worn dentition can be accomplished with full or partial coverage restorations. ${ }^{2}$ In recent years, new dental materials and techniques in combination with advances in dental adhesion were developed to enable clinicians to perform conservative approaches. ${ }^{5}$ In the present case, partial coverage restorations as laminate veneers for anterior teeth and vonlays for posterior teeth were applied on maxilla. These restorations enabled maximum preservation of sound tooth structure with minimal tooth reduction that provide adequate restorative space for lithium disilicate glass-ceramic restorative material instead of traditional full-coverage preparations that require excessive hard tissue removal. Therefore, in this case, vonlay restorations were proved to combine esthetic and conservative approach of veneer and onlay restorations as an alternative to crown restoration. ${ }^{13}$ In generalized severe toothwear cases, the loss of OVD is a frequently encountered problem. ${ }^{14}$ Restoring functional occlusal relationships with the reconstruction of OVD requires a complex, multistep treatment. ${ }^{15}$ The increase in OVD can be provided by one- or twostep approaches. The one-step approach employs a treatment modality that the definitive indirect restorations being all bonded within the same or following day, whereas provisional restorations or nightguards are applied to test the new

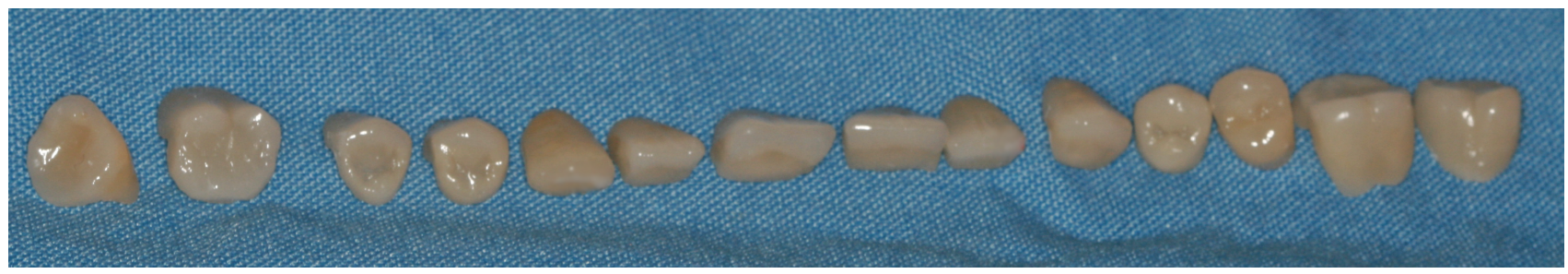

Figure 6. Maxillary lithium disilicate restorations 


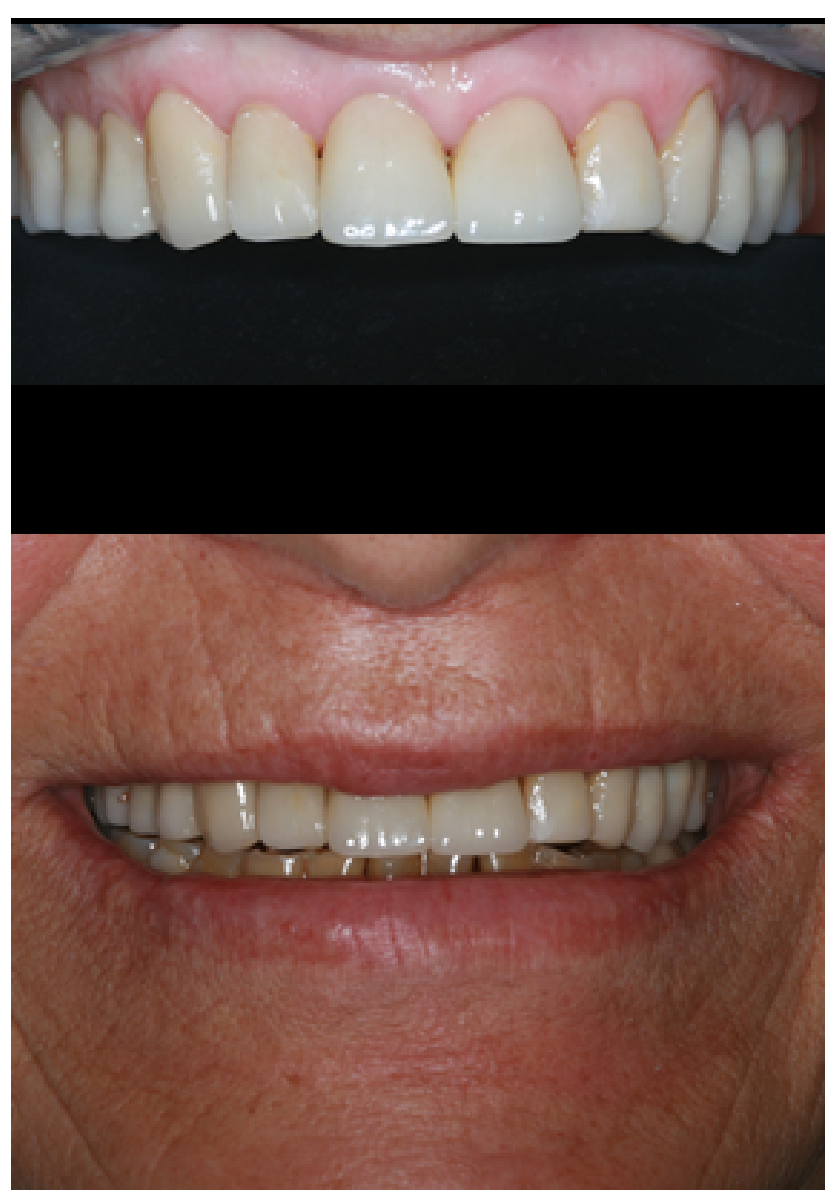

Figure 7. Final result of the patient

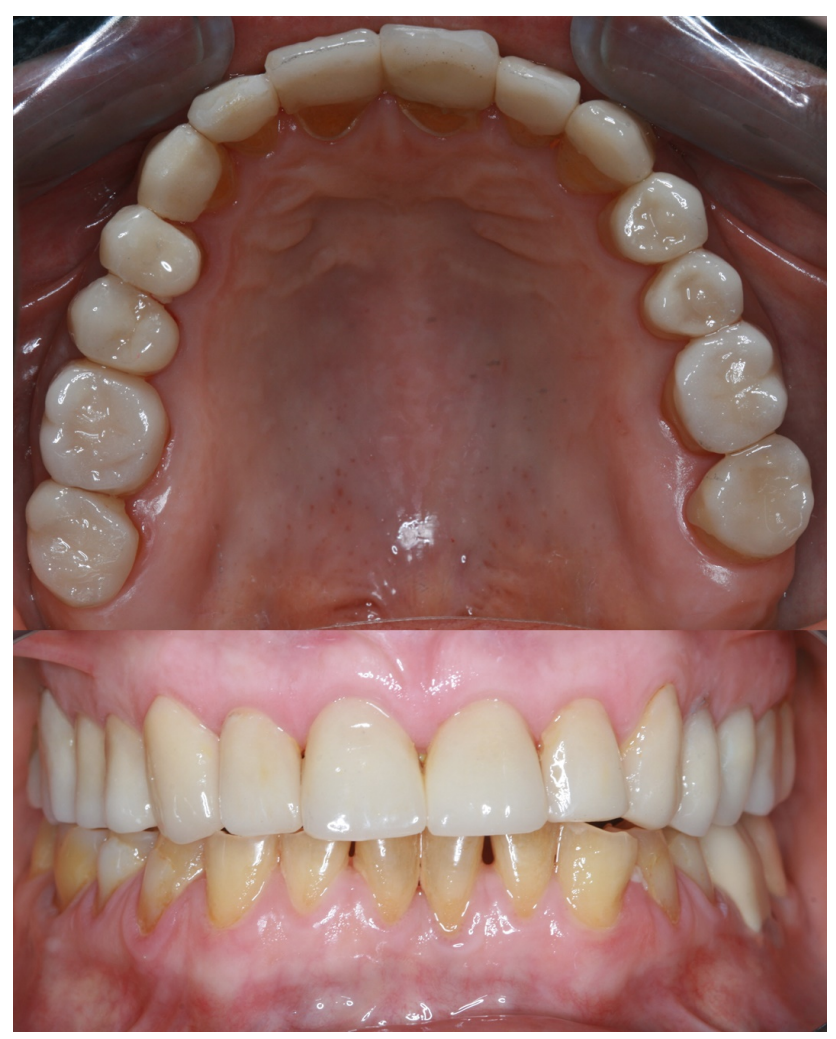

Figure 8. Intraoral photographs of the definitive restorations
OVD with the two-step approach. ${ }^{12}$ The clinical indication for choosing between these two approaches is the amount of required OVD increase. ${ }^{12}$ Previous studies reported that an increase in OVD up to $5 \mathrm{~mm}$ in the maxillo-mandibular interincisal distance is well tolerated by patients and that one-step approach is applicable. ${ }^{15,16}$ On the other hand, OVD increase in patients with severe attrition induces signs of neuroplastic changes in corticomotor control of masseter muscles. ${ }^{17}$ Therefore, when alteration in OVD is planned for patients with TMD, two-step approach is suggested regardless of the amount of increase. ${ }^{10}$ The patient in the present case stated a dubious dental anamnesis about TMD-associated symptoms. Therefore, the increase in OVD was tested by provisional direct resin composite restorations before any form of irreversible procedure.

Bruxism is a parafunctional activity of the masticatory system which induces increases in the maximum bite force. ${ }^{18}$ For individual subjects with bruxism, Nishigawa et al. ${ }^{19}$ reported that mean maximum voluntary and nocturnal bite force may increase as high as 774.73 and 414.82 Newton $(\mathrm{N})$, respectively. However, the mean fracture resistance for lithium disilicate glass ceramic vonlays applied on premolars was reported as $582.3 \mathrm{~N} .{ }^{13}$ Since the signs of bruxism were evident for our patient, employing a night-guard to preserve restorations was crucial. Hence, immediately after the cementation of the final restorations, an alginate impression was taken, and a vacuumformed occlusal splint was delivered to the patient within the same day. The patient was warned to wear it every night.

\section{Conclusion}

Rehabilitation of severely worn dentition requires consideration based on not only functional occlusion, but also esthetic appearance. This case report described the treatment process of a worn maxillary dentition with esthetic laminate veneer and vonlay restorations by establishing a new OVD. In such cases with comprehensive treatments, the evaluation period, provisional and definitive restorations, and the maintenance phase should be planned carefully.

\section{Author Contributions}

Case planning and Methodology: EIO, SB. Writing - Original Draft Preparation Creation and/or presentation of the published work: EIO. Writing - Review \& Editing: SB. Both authors reviewed the results and approved the final version of the manuscript.

\section{Conflict of Interest}

Authors declare that they have no conflict of interest.

\section{Authors' ORCID(s)}

E.I.O. $\quad 0000-0001-6128-9723$

S.B. $0000-0003-0731-4750$

\section{References}

1. Alqahtani F. Full-mouth rehabilitation of severely worn dentition due to soda swishing: a clinical report. J Prosthodont. 2014;23(1):50-7. doi:10.1111/jopr.12097. 
2. Maroulakos G, Nagy WW, Stathopoulou PG, Mattheos N, Chronopoulos V. Restoration of the Worn Dentition: Basic Prosthodontic Principles and Current Rehabilitation Techniques. Curr Oral Health Rep. 2017;4(2):124-130. doi:10.1007/s40496-017-0133-3.

3. Van't Spijker A, Rodriguez JM, Kreulen CM, Bronkhorst EM, Bartlett DW, Creugers NH. Prevalence of tooth wear in adults. Int J Prosthodont. 2009;22(1):35-42.

4. Vailati F, Belser UC. Full-mouth adhesive rehabilitation of a severely eroded dentition: the three-step technique. Part 1. Eur J Esthet Dent. 2008;3(1):30-44.

5. Bahillo J, Jané L, Bortolotto T, Krejci I, Roig M. Fullmouth composite rehabilitation of a mixed erosion and attrition patient: a case report with $\mathrm{v}$-shaped veneers and ultra-thin CAD/CAM composite overlays. Quintessence Int. 2014;45(9):749-56. doi:10.3290/j.qi.a32439.

6. Kaidonis JA, Richards LC, Townsend GC, Tansley GD. Wear of human enamel: a quantitative in vitro assessment. J Dent Res. 1998;77(12):1983-90. doi:10.1177/00220345980770120601.

7. Al-Salehi SK. Restorative Management of Intrinsic and Extrinsic Dental Erosion. J Indian Prosthodont Soc. 2014;14(Suppl 1):215-21. doi:10.1007/s13191-013-0274-6.

8. Bettie NF, Kandasamy S, Prasad V. Management of Tooth Surface Loss of Varying Etiology with Full Mouth all Ceramic Computer-Aided Design/Computer-Aided Manufacture Restorations. J Pharm Bioallied Sci. 2017;9(Suppl 1):S302-s305. doi:10.4103/jpbs.jpbs_130_17.

9. Sudhir N, Chittaranjan B, Kumar BA, Taruna M, Kumar MP, Reddy MR. Digital Cephalometric Tracings by PROCEPH V3 Software for Comparative Analyses of Vertical Dimension in Edentulous Patients. J Clin Diagn Res. 2015;9(5):Zc01-5. doi:10.7860/jcdr/2015/12034.5862.

10. Abduo J, Lyons K. Clinical considerations for increasing occlusal vertical dimension: a review. Aust Dent J. 2012;57(1):2-10. doi:10.1111/j.1834-7819.2011.01640.x.

11. Alothman Y, Bamasoud MS. The Success of Dental Veneers According To Preparation Design and Material
Type. Open Access Maced J Med Sci. 2018;6(12):2402-2408. doi:10.3889/oamjms.2018.353.

12. Oudkerk J, Eldafrawy $M$, Bekaert S, Grenade C, Vanheusden A, Mainjot A. The one-step no-prep approach for full-mouth rehabilitation of worn dentition using PICN CAD-CAM restorations: 2-yr results of a prospective clinical study. J Dent. 2020;92:103245. doi:10.1016/j.jdent.2019.103245.

13. Elsayed M, Sherif R, El-khodary N. Fracture resistance of Vita suprinity versus IPS e. max CAD vonlays restoring premolars (An in vitro study). Int J Appl Dent Sci. 2020. doi:10.22271/oral.2020.v6.i3k.1029.

14. Mehta SB, Banerji S, Millar BJ, Suarez-Feito JM. Current concepts on the management of tooth wear: part 1 . Assessment, treatment planning and strategies for the prevention and the passive management of tooth wear. Br Dent J. 2012;212(1):17-27. doi:10.1038/sj.bdj.2011.1099.

15. Mainjot AKJ. The One step-No prep technique: A straightforward and minimally invasive approach for full-mouth rehabilitation of worn dentition using polymer-infiltrated ceramic network (PICN) CAD-CAM prostheses. J Esthet Restor Dent. 2020;32(2):141-149. doi:10.1111/jerd.12432.

16. Ormianer Z, Palty A. Altered vertical dimension of occlusion: a comparative retrospective pilot study of tooth- and implant-supported restorations. Int J Oral Maxillofac Implants. 2009;24(3):497-501.

17. Deng H, Gao S, Lu S, Kumar A, Zhang Z, Svensson P. Alteration of occlusal vertical dimension induces signs of neuroplastic changes in corticomotor control of masseter muscles: Preliminary findings. J Oral Rehabil. 2018;45(9):710719. doi:10.1111/joor.12682.

18. Todić JT, Mitić A, Lazić D, Radosavljević R, Staletović M. Effects of bruxism on the maximum bite force. Vojnosanit Pregl. 2017;74(2):138-144.

19. Nishigawa K, Bando E, Nakano M. Quantitative study of bite force during sleep associated bruxism. J Oral Rehabil. 2001;28(5):485-91. doi:10.1046/j.13652842.2001.00692.x. 